\title{
On If and Only
}

\author{
Paul Dekker \\ ILLC/University of Amsterdam \\ dekker@hum . uva.nl
}

Possibly the interpretation of the lexical items "if" and "only" is the subject disputed most in the largest number of language oriented disciplines, such as logic, natural language semantics, cognitive psychology and artificial intelligence. In this paper I want to focus on the logical and linguistic aspects of these items. I do not aim to present a brand new theory about or new insights on the two subjects. My aim, rather, is to try and improve upon existing analyses and where possible help with integrating them.

Some standard formal and logical analyses of "if" and "only" are very attractive from a theoretical point of view, but their compatibility with more empirically oriented findings about the use of "if" and "only" in natural language can be questioned. With this paper I want to support the view that no such incompatibilities exist, that we witness two ways of approaching the same phenomenon, and that the findings in one perspective may contribute to those obtained in the other.

As a starting point I take a system of dynamic semantics, actually my mother tongue, formally speaking. I will point out that a rigid dynamic semantic conception of meaning as such stands in the way of a more general, flexible, notion of interpretation, including that of if-clauses (section 1). I will next study a more empirically motivated interpretation of if-clauses as domain restrictors and argue that this alternative interpretation does not really pay off (section 2). In section $3 \mathrm{I}$ sketch an analysis of ordinary, inverse, and only if-donkey sentences on the basis of (i) a classical notion of implication, (ii) a dynamic semantic notion of information, and (iii) an independently needed notion of information structure. In section 4 these more or less theoretical observations are tested on a number of only if-donkey sentences which I collected on the internet. My basic intuitions seem to be supported by this little corpus, although they are not sufficient to account for all of the data. Section 5 gives a glimpse into the reasons why I think the basic interpretation of "if" and "only" should not be taken to account for these additional data-mainly because these can be attributed to (the interplay with) other modal and pragmatic aspects of the conditionals at issue.

\section{Dynamic Semantics (Second Generation)}

One of the major achievements of the dynamic semantic paradigm is its uniform semantic account of anaphoric relationships across conjunctions and implications. Let us focus in on a dynamic semantics like that of dynamic predicate logic (Groenendijk and Stokhof 1991, DPL), by and large inspired by discourse representation theory (Kamp 1984; Kamp and Reyle 1993 DRT) and file change semantics 
(Heim 1982 FCS). The characteristic (distinctive) feature of $D P L$ is that it renders (1, 'Egli's theorem') semantically valid:

(1) $(\exists x \phi \wedge \psi(x)) \Leftrightarrow \exists x(\phi \wedge \psi(x))$

By means of the supporting dynamic semantics, a formal and systematic analysis has thus been provided of a couple of sentences that have troubled semantic theory for ages. The equivalence in (1) reflects the intuitive, truth-conditional, equivalence of the following two examples:

(2) A man is walking in the park. He is whistling.

(3) A man who is walking in the park is whistling.

The core (dynamic semantic) idea behind the equivalence in (1) has always been that (i) meaning is some kind of update potential and (ii) indefinites 'introduce' some kind of discourse referents, which are available for being picked up by subsequent anaphoric pronouns.

Quite an appealing feature of $D P L$ (and of $D R T$ and $F C S$, if you want) is that it renders (4) valid, as a mere consequence of (1):

(4) $(\exists x \phi \rightarrow \psi(x)) \Leftrightarrow \neg(\exists x \phi \wedge \neg \psi(x)) \Leftrightarrow(1)$ $\neg \exists x(\phi \wedge \neg \psi(x)) \Leftrightarrow \forall x(\phi \rightarrow \psi(x))$

Thus, DPL (like related systems) is able to deal, successfully, with Geach's notorious donkey-sentence (5) which has standardly been taking to involve universal quantification over farmer-donkey pairs:

(5) If a farmer owns a donkey (s)he beats it.

Every farmer beats every donkey he or she owns.

Example (5) has it that if you are presented with a (any) farmer who owns a (any) donkey, you will find that the first beats the latter.

From a formal point of view DPL is very well behaved: its semantics is elegant and transparent, and it comes with a nice deduction system (Veltman 2001, cf., also, van Eijck 2001). However, as a model for the interpretation of anaphoric relationships in natural language it is rather limited and, worse, too rigid to be suitably extended. The reason is that a notion of update is hardwired into the system's notion of conjunction (and that of implication) and that anaphoric pronouns are associated with possible referents which can not but have the properties attributed to their antecedents. ${ }^{1}$ This is problematic, as can be seen from Strawson's famous example of pronominal contradiction:

(6) A: A man just fell of the cliff.

(7) $B:$ He didn't fall, he was pushed.

In this example person $B$ denies the referent to have the property previously ascribed to him by $A$. Besides, $B$ even might continue with:

(8) $B$ : Besides, it was a woman.

In defense of an update (or E-type) approach one might claim that Strawson's example is a special case, involving an intended referent which is (or, rather: was) 
demonstratively present. As argued elsewhere (e.g., Dekker 2001) we do not think demonstrative presence is a prerequisite for cross speaker anaphora such as we might witness here, and, besides, it may be fairly obvious that someone who overhears Strawson's protagonists may very well understand what they claim without having access to the individual $A$ and $B$ intended to refer to (and which may even be the product of their imagination).

Rigid update systems of interpretation are also deemed to fail in response to minor variants of (5) such as:

(9) A farmer may beat a donkey if (s)he owns it.

(10) Only if a farmer owns a donkey may (s)he beat it.

In example (9) the clause "a farmer beats a donkey" is semantically (conditionally) dependent on the phrase "he owns it", whereas the latter is structurally (anaphorically) dependent on the first. In a one-dimensional update system of interpretation this constitutes a paradox of interpretation. Essentially the same goes for example (10). The only if clause describes a necessary condition for a situation as described by the main clause to obtain. But what would such a situation be, according to the update analysis of pronouns? That (s)he, a farmer who owns a donkey, may beat it, the donkey (s)he owns? But if this is indeed the situation at issue, (10) would be vacuously true-which, as a matter of fact, the sentence is not. ${ }^{2}$

The need for a potentially more flexible treatment of anaphoric relationships has given rise to what may be termed 'second generation' systems of dynamic semantics. These systems crucially employ the notion of information developed in the first generation (Heimian information sets, say, the semantic correlate of discourse representation structures). The crucial difference with the first generation is that sentence meanings are not taken to be updates of these information sets, but information sets themselves. By means of a dynamic notion of conjunction, which is derived from a classical notion of conjunction as intersection, anaphoric relationships between indefinites and anaphoric pronouns can be established in a flexible way.

The system underlying the remainder of this paper is such a system 'predicate logic with dynamic conjunction' (PLDC, Dekker 2000). ${ }^{3}$ This system is by and large inspired by (Stalnaker 1978; Stalnaker 1998) and close in basic spirit to that of (Zeevat 1989; van der Does 1996; van Rooy 1997).

Passing over all technical details, Egli's theorem (1) is also valid in PLA, as is the donkey equivalence (4). However, since the dynamics resides in a specific form of conjunction, and since it is not inherently encoded in the meanings of the sentences themselves, it is easy to come up with other forms of merging information sets. In particular, the information sets associated with Strawson's examples (6) and (7) can be construed as being (intended to be) about one individual, without this requiring us to merge their contents.

The nice thing about a system like PLDC then is, that, on the one hand, it is more flexible in easily allowing other forms of merging information, whereas, on the other hand, it comprises $D P L$ as a special case. This indeed implies that the nice 
logical properties of $D P L$ can be preserved, be it that they are qualified as logical properties in a restricted domain of application: that of monologue, or indeed of dialogue without conflict and revision.

Having given a very rough sketch of the basic machinery, let us turn back to "if" and "only". In this paper we will assume basic interpretations of "if" and "only" in the spirit of Philo, Frege, Quine and Geach (but also Peirce and Grice) on the one hand, and a variety of authors like Horn, Rooth, de Mey and Hendriks, (among many many others) on the other. The basic idea is to associate "if" with $\rightarrow$ (and, more generally, with $\subseteq$ ), and "only" with $\supseteq$ (and, in specific cases, with $\leftarrow$ ). ${ }^{4}$ Simple motivating examples are the following:

(11) Who qualify?

- PostDocs do. $(P \subseteq Q)$

- Only PostDocs do. $(P \supseteq Q)$

(12) Will Francis get the job?

- If he satisfies the prerequisites. $(p \rightarrow q)$

- Only if he satisfies the prerequisites. $(p \leftarrow q)$

A more general interpretation is required in the following setting:

(13) When does a student qualify?

- If (s)he satisfies the prerequisites. $(p \subseteq q)$

- Only if (s)he satisfies the prerequisites. $(p \supseteq q)$

Before we take a further look at the inverse and only if donkey sentences (9) and (10), let us first consider an alternative idea about the meaning of if-clauses, which has gained some popularity in the literature.

\section{If-Clauses as Domain Restrictors?}

Quite a few authors have observed that if -clauses also figure as 'domain restrictors' (Lewis 1975; Heim 1982; Kratzer 1991; von Fintel 1998), and it has even been argued that domain restriction is their pivotal role, cf., e.g.:

"The history of the conditional is the story of a syntactic mistake. There is no two-place if... then connective in natural language. If -clauses are devices for restricting the domains of various operators." (Kratzer 1991, p. 656)

Such a conclusion is by and large motivated by sentences with adverbial quantifiers, in which the if-clause indeed provides the restriction of an adverbial quantifier, and in the which main clause figures as its nuclear scope:

(14) If a friend has problems Joe sometimes/usually/always/never helps him out. 
Intuitively these examples seem to express that Joe helps in $\{$ some / most / all / no cases in which a friend has problems.

It has also been argued that if-clauses may serve to impose further constraints on the restriction of adnominal quantifiers. Consider the following two examples due to (Higginbotham 1986) : $^{2}$

(15) Every student will succeed if he works hard.

(16) No student will succeed if he goofs off.

Example (15) can be given an intuitively correct interpretation, if it is taken to state that for every student the following holds: if he works hard he will succeed. However, a similar analysis of example (16) would give rather disastrous results. For suppose example (16) is rendered as stating that for no student this holds: if he goofs off he will succeed. If we read the latter implication as a material one, then the sentence would turn out to state that every student goofs off and no student succeeds, which is way too strong. Alternatively, if the sentence is taken to state that for no student there is a rule-governed connection between goofing off and success, then this is way too weak. Rather, example (16) seems to state that no student who goofs off will succeed, that is, that goofing off implies failure.

Intuitively acceptable readings of both (15) and (16) can be obtained if the two if-clauses are interpreted as constraining the quantifier's restriction. Example (15) says that every student who works hard succeeds and example (16) that no students who goofs off succeeds. Something essentially similar holds of two examples which Kai von Fintel has attributed to Irene Heim:

(17) Few people like New York if they didn't grow up there.

(18) Most letters are answered if they are shorter than 5 pages.

Intuitively, (17) states that few people who didn't grow up in New York like New York, and example (18) can be taken to state that most letters which are shorter than five pages are answered. Finally, the following examples can be interpreted in a similar way:

(19) Students qualify if they work hard.

Example (19) intuitively says that hard working students qualify. The case for a domain restriction analysis of if thus appears to be rather strong. Indeed:

"Thus there are good reasons to adopt the restrictor analysis for noun-phrase indicatives and not just for sentences involving adverbial quantifiers." (von Fintel 1998, p. 212)

The case is not that simple, though, as the following discussion may serve to show.

First, consider some slight modifications of the students' success stories:

(20) Derek succeeds if he works hard.

There seems to be nothing to restrict in (20), and certainly it does not say that among those who work hard, Derek succeeds. Rather, the interpretation of (20) must involve some implication, that hard work will guarantee success for Derek. 
(21) Only Derek succeeds if he works hard.

(22) Only students succeed if they work hard.

A domain restriction reading of (21, if any) and (22) would entail that all who succeed work hard, whether they are students (or Derek) or not. Instead, on the wide scope only reading the sentences seem to state something different. ${ }^{6}$ The sentences then can be taken to state that if someone succeeds who has worked hard, it is Derek, or a student,respectively.

The domain restriction analysis goes even more drastically astray when we consider examples with indefinites and numeral determiners.

(23) Some students succeed if they work hard.

(24) Exactly three students succeed if they work hard.

Clearly, (23) and (24) do not serve to state that some / exactly three students who work hard succeed. These sentences instead say something about the number of students for which hard work is a guarantee for success. An implication analysis of these sentences thus seems to be most appropriate, provided that the domain of quantification is restricted to those students for which (to the speaker's knowledge) working hard is still an option. ${ }^{7}$

To conclude this negative part, let us finally point out another rather severe complication for a domain restriction analysis. Consider:

(25) Every student accepts any position if (s)he qualifies for it.

(26) No postdoc disregards a job opportunity if (s)he qualifies for it.

If if-clauses constrain the domains of quantifiers, then they must be taken to constrain two domains simultaneously in (25) and (26). This is not impossible, but it certainly transcends the capacities of the envisaged techniques. Instead, it seems that for these examples, as for the examples (20-24), some implication analysis of a main-clause if-subordinate clause structure is appropriate. But then, again, what about (15-19)? Actually, an implication analysis of these sentences does not fare that bad at all.

First observe that an analysis of if $A$ as $\lambda q p \rightarrow q$ is appropriate for (15) (and 25) and (19). For if any hard working student qualifies, then, and only then, it is true for any student that (s)he qualifies if (s)he works hard. Schematically:

(27) $(S \cap W H) \subseteq Q$ iff $S \subseteq(\overline{W H} \cup Q)$

With respect to the examples (16), and (17) (and 22) observe that the respective verb phrases figure in a downward entailing context. In (Dekker 1993; Dekker 1999, Ch. 3) I have argued that external modification of material in a downward entailing context is subject to a dualization operator *, which guarantees preservation of monotonicity properties. Such a logical operator turns a conditionalizer $\lambda q p \rightarrow q$ into a conjoiner $\lambda q p \wedge q$ :

(28) $(\lambda q p \rightarrow q)^{*}=\lambda q \neg(p \rightarrow \neg q)=\lambda q p \wedge q$ 
If used in the interpretation of example (16) the example will be read as stating that no student (will) goof off and succeed, (17) as stating that few people didn't grow up in New York and like the city, and (22) as stating that only students both work hard and succeed. Surely, there are some modal and aspectual properties which are thus not accounted for (and which we will come back to below), but truthconditionally these paraphrases are equivalent with those obtained on the domain restriction reading.

This leaves us with example (18):

(18) Most letters are answered if they are shorter than 5 pages.

Also for this example an implicative reading is certainly not inappropriate. Example (18) can be taken to say that most letters are subject to the rule or principle that if a letter is shorter than 5 pages, then it gets answered. ${ }^{8}$ This reading is slightly different from the one in which the if-clause figures as a domain restrictor. For instance, suppose 5 letters are still under consideration, 3 are shorter than 5 pages and one of the three is answered. In that case 3 out of 5 letters (the answered letter and the 2 longer ones) obey the principle so the sentence would be true under the implicative reading. In the same case only 1 out of 3 letters which are shorter than 5 pages are answered so the sentence would be false under the restrictive reading. I leave it to the general public to judge the issue.

So far we have argued that indeed there is nothing obviously wrong with an implicative interpretation of if-clauses, and that a domain restrictive reading is not generally tenable. If -clauses do not restrict adnominal quantifiers. But what about the adverbial quantifiers, which made up the clearest motivation for domain restriction analyses? Here we have to point out that, if, indeed, if-clauses impose conditions on the domains of quantification of adverbial quantifiers, this does *not* mean they are not $\lambda q p \rightarrow q$, basically. Suppose $p$ is an (intentionally closed) proposition, a condition on worlds, situations, assignments, sequences of individuals, or what have you. Suppose, moreover, we analyze an if $A$-clause as ${ }^{\wedge} \lambda q\left({ }^{\vee} p \rightarrow{ }^{\vee} q\right)$, abbreviated as $R$. The condition $p$ then can be retrieved from $R$ in the following way:

(29) $E(R)={ }_{d f} \wedge \forall q\left({ }^{\vee} R(q) \rightarrow{ }^{\vee} q\right)$

With $R$ as specified, this gives us $\wedge^{\wedge} \forall q\left(\left({ }^{\vee} p \rightarrow{ }^{\vee} q\right) \rightarrow{ }^{\vee} q\right) \Leftrightarrow{ }^{\wedge} \forall q\left({ }^{\vee} p \vee{ }^{\vee} q\right)$. Indeed, the worlds (situations, ...) in which $p$ is true in disjunction with any proposition, including a false one, is precisely the set of worlds (situations, ...) in which $p$ is true. $^{9}$ As this result is perfectly general, we think we can be satisfied here with not choosing between analyzing if -clauses as restrictive or as implicative. ${ }^{10}$ Notice, again, that, since we can retrieve $p$ from $\lambda q p \rightarrow q$, the meaning of only if $p$ ( $\lambda q p \leftarrow$ $q)$ can be defined as a function on the meaning of if $p$.

To conclude this section, let us sum up the results. We have argued that if-clauses do not generally restrict adnominal quantifiers, that they may restrict that of adverbial (or adsentential) quantifiers, but that they can do so also on their $\lambda q p \rightarrow q$ interpretation. Finally, we have observed some presuppositional restrictions upon domains of quantification, which will be taken more serious in the next section. 


\section{Inverse and Only If Donkey Sentences}

Let us now turn back to inverse and only if-donkey sentences. In (von Fintel 1994) it has already been observed that phonological structure (with associated information structure) is highly relevant for the interpretation of inverse and only if-donkey sentences. Consider:

(30) A farmer may [beat $]_{F}$ a donkey if (s)he owns it.

(31) Only if a farmer [owns $]_{F}$ a donkey may (s)he beat it.

These examples seem to be fine. They appear to be about farmers and donkeys, and either the question when a farmer is allowed to beat a donkey, or the question what a farmer may do to a donkey he owns. Of all pairs of a farmer and a donkey (30) says that if it is an own-pair, it is also a may-beat-pair, and (31) that only if it is an ownpair it is a may-beat-pair. The farmers and donkeys, in other words, seem to have escaped the conditional structures, and to live in their domain of quantification. A proper interpretation of the pronouns thus seems to be possible precisely because they relate to an antecedent which, semantically, is not dependent on the clause which the pronoun figures in. The whole conditional structures, with the pronouns in them, are dependent on the domains of quantification evoked by "a farmer" and "a donkey".

Such an analysis can be motivated further by examples where some of the indefinites figure in a focalized constituent:

(32) ? A farmer may [beat a donkey $]_{F}$ if (s)he owns it.

(33) ? Only if a farmer [owns a donkey $]_{F}$ may (s)he beat it.

These sentences are problematic, semantically speaking. They appear to be about farmers and to address the question when such a farmer is allowed to beat a donkey. But, if this is so, then it is unclear what the pronoun "it" stands for. It is clear that the pronoun "he" in these examples, as in the previous ones, stands for a, or any, farmer. But where "it" in the previous examples clearly refers to a or any donkey, it seems to be unresolved here. "Only if a farmer [owns a donkey $]_{F}$ may he beat what?" seems to be a natural response to (33). Again, a reply with "a, or any, donkey he owns" would render the example trivial. The examples are odd, we claim, because the donkeys are focalized, and they are therefore unable to escape the conditional structure. Putting it the other way around, indefinites may escape certain semantic structures, and figure as antecedents of subsequent anaphoric pronouns only if they are non-focal (non-novel, or "topical" as we will also say). This observation is not at all new by the way (cf., e.g., Gawron 1996; Aloni et al. 1999; Krifka 2001), but (30-33) provide striking examples of the phenomenon.

Before we turn to a (sketch of an) analysis, consider the two replies in example (13) again. They could as well have been formulated as follows:

(34) A student qualifies if (s)he satisfies the prerequisites. If a student satisfies the prerequisites (s)he qualifies. 
(35) A student qualifies only if (s)he satisfies the prerequisites.

Only if a student satisfies the prerequisites (s)he qualifies.

As a reply to the question in (13) it is clear that these statements are about the students and about the question whether or in which cases they qualify. Notice that it is immaterial whether the indefinite is in the main or in the subordinate clause in (34) and (35). Thus, the semantic contribution of these topical phrases indeed seems to escape from the structures in which they figure.

Let us finally point at a parallel mechanism which can be seen to be at work in the following examples, the first attributed to Marco Bikker by Helen de Hoop, the second from Regine Eckardt:

(36) Most boys were rejected because of their height.

(37) Almost all tickets were sold at checker 4.

(38) Only boys who were rejected because of their height may file a complaint.

Example (36) can be taken to quantify over the boys who were rejected. Similarly, (37) can be understood as an assertion about the tickets which were sold, and (38) as an assertion about boys who were rejected (and who may file a complaint). As in the previous examples, non-focal material figuring at one place in the structures-in the verb phrase in (36-37) and in a restrictive relative clause in (38)-escapes from there and somehow projects itself into a domain of quantification. In the remainder of this section we will see how this behavior can be modeled in a straightforward way using techniques dealing with presupposition and information structure familiar from the literature.

In line with most, if not all, theories of presupposition and information structure we assume that information conveyed by means of natural language can be divided into a presupposition or ground part, and an assertion or focal part. More specifically, we will assume some compositional method of building such structured pieces of information in the style of (Karttunen and Peters 1979), using Heimian information sets to model first order information. ${ }^{11}$

In all of the examples which we have discussed in this section, presuppositionallike material restricts a domain of quantification. In principle, this type of domain restriction can be effectuated in two ways. Presuppositions may impose additional constraints on the explicitly specified restrictions of quantifiers, as on the domain restriction analysis of if-clauses, or as constraining the contextually given domain of quantification (cf., Westerståhl 1984). ${ }^{12}$ It seems the latter option has to be preferred, intuitively, but, arguably, also empirically.

First observe that contextually restricted domains of quantification should be kept distinct from the first argument of a non-conservative term like only (examples like these are discussed by (Jäger 1996), and by Herman Hendriks, in many presentations):

(39) Which Athenians are wise?

(40) Only sophists are wise. $\left(O_{A}(S)(W) \Leftrightarrow(A \cap S) \supseteq(A \cap W)\right)$ 
As a reply to (39), (40) can be used to state, not that all wise men are Athenian sophists, but, rather, that only (Athenian) sophists are wise Athenians. Similarly, example (38) has a reading which entails nothing about girls or about boys who were not rejected. This reading can be obtained if the non-focal condition of being a boy who is rejected is projected, from the restriction of only into the domain of quantification. We take it then, that such presuppositions, thus, restrict the domain of quantification and do not constrain the restriction of the determiner.

Let us now try and see how the Bikker - de Hoop - Eckardt type of examples can be handled. Presuppositional restriction of domains of quantification can be represented, roughly, as:

(41) $G Q_{C}(A)(\langle B ; D\rangle) \Leftrightarrow G Q_{C}(\langle B ; A\rangle)(D) \Leftrightarrow G Q_{C \cap B}(A)(D)$

Backgrounded material $B$ in the nuclear scope or in the restriction of a quantifier $G Q$ can simply be used to constrain the (possibly presupposed) domain of quantification $C$. For (37) and (38) this gives us the intended readings:

(37) Almost all tickets were sold at checker 4.

$$
A A_{C}(T)(\langle S ; S A T 4\rangle) \Leftrightarrow A A_{C \cap S}(T)(S A T 4)
$$

(38) Only boys who were rejected because of their height may file a complaint. $\left.O_{C}(\langle B \cap R ; R B O T H\rangle)(M F C) \Leftrightarrow O_{C \cap B \cap R}(B \cap R B O T H)(M F C)\right)$

Example (37) is evaluated against a background domain of quantification consisting of the tickets which were sold (in the present context $C$ ). Example (38) relates to the boys (in the context) who were rejected, and it is taken to state that only those among them who were rejected because of their height may file a complaint.

Essentially the same techniques can be used to model what is going on in inverse and only if-donkey sentences, now we have the means to deal with anaphoric relationships in a sophisticated way. In the following examples it is important to realize that context sets, like information sets, may contain information about tuples of individuals:

(30) A farmer may [beat $]_{F}$ a donkey if (s)he owns it.

$$
\begin{aligned}
& I I_{C}(\langle\exists x(F(x) \wedge \exists y D(y) ; M B(x)(y)\rangle)(O(x)(y)) \Leftrightarrow \\
& \forall(x, y)_{C}((F(x) \wedge D(y)) \rightarrow(M B(x)(y) \leftarrow O(x)(y)))
\end{aligned}
$$

Example (30) is true iff for all pairs of farmers $x$ and donkeys $y$ in the context this holds: $x$ may beat $y$ if $x$ owns $y$.

(31) Only if a farmer [owns $]_{F}$ a donkey may (s)he beat it. $O I_{C}(\langle\exists x(F(x) \wedge \exists y D(y) ; O(x)(y)\rangle)(M B(x)(y)) \Leftrightarrow$ $\forall(x, y)_{C}((F(x) \wedge D(y)) \rightarrow(O(x)(y) \leftarrow M B(x)(y)))$

Example (31) is true iff for all of the same type of pairs: only if $x$ owns $y, x$ may beat $y$. Interestingly, the very same type of analysis seems to be fit for ordinary donkey-sentences:

(5) If a farmer [owns $]_{F}$ a donkey (s)he beats it.

$$
\begin{aligned}
& I_{C}(\langle\exists x(F(x) \wedge \exists y D(y) ; O(x)(y)\rangle)(B(x)(y)) \Leftrightarrow \\
& \forall(x, y)_{C}((F(x) \wedge D(y)) \rightarrow(O(x)(y) \rightarrow B(x)(y)))
\end{aligned}
$$


Such a donkey sentence is rendered true iff for all of the same type of pairs above: if $x$ owns $y$ then $x$ beats $y$. This is interesting, because it shows we get a strong reading of donkey sentences, not just from a dynamic semantic interpretation of $\rightarrow$, but from a basically classical interpretation of $\rightarrow$ and independently motivated principles of interpreting information (anaphoric) structure. In short, all key examples motivating a dynamic semantics can be handled using a static semantics, an independently motivated notion of information structure, a flexible combinatorics, and, not unimportantly, a dynamic semantic notion of information. ${ }^{13}$

In this section we have sketched an analysis of inverse and only if -donkey sentences in terms of their information structure, and based on the independently motivated idea that topical or non-focal material projects into domains of quantification. The analysis generalizes to ordinary donkey sentences and allows us to do without a dynamic or $D R T$-style analysis of $\rightarrow$ and stick to a classical one.

\section{Only if a Internet Search}

Some semantic/linguistic discussions tend to get so sophisticated that they reach a realm of subtle expert examples and intuitions which do not raise any sensible reaction (support, rejection) from ordinary language users. For this reason I have tried to lift our arm-chair philosophy to the level of mouse-arm linguistics. I was seriously interested in the question if only if-donkey sentences are actually used, and, besides, I must admit, it was easy enough to look for sentences like this with an ordinary search engine. I was pretty much surprised to find an interesting lot of these examples, and in this section I will comment upon them from the perspectives sketched in the previous sections. I hope it needs no comments that my findings in this section are statistically not at all significant. I have not collected a sufficiently significant corpus of examples, and my evaluation of each of the examples is not tested upon judgements of others.

I did my search with the search engine Altavista, 2000 October 10, 11.35, looking for +"Only if a ". Altavista reported:

(42) "1968 pages found", "word count: Only if a: 320"

and presented me 200 hits. One third of these were misfires, so I ended up with a corpus of 133 examples. ${ }^{14}$ Not all examples were really understandable as such, e.g.:

(43) June 12, 2000 - What should Ollie do?

$47 \%$ Yes, in every case

$48 \%$ No, never

$5 \%$ Only if a repeat offender [128]

(1) Like I said, the humble question which I started out with was:

- are only if-donkey sentences actually used? 
and the answer is unconditionally positive. In more than half of the examples an indefinite in the if-clause is anaphorically related to material in the main clause, directly (as in (44)) or more indirectly (as in (45)):

(44) Only if a greenish beryl is found without either a yellow or blue tone will it be called a "green" beryl. [6]

(45) Only if a project takes an extremely excessive amount of time and work will the fee be any more than that. [1]

(2) In almost all of these examples the indefinite(s) can be seen to restrict the domain of quantification. Sometimes the indefinites are partly topical (as in (46)) or functional upon the things that actually are quantified over (as in (47)):

(46) Only if a "plain vanilla" ISO-9660 CD were being burned would these messages be of any interest, and such a CD would be useless for Linux installation anyway. [47]

(47) Only if a second smoke detector trip occurs within a short time, typically 60 seconds, or if the detector fails to reset, an alarm is activated [34]

Only seven examples are, arguably, one-case only if-conditionals, such as (48):

(48) Only if a miracle takes place will the far reaching goals it set at the start of the decade be reached. [18]

(3) Generally only if-clauses express necessary conditions for the condition in the main clause. There is only one obvious exception in our corpus:

(49) Only if a trick contains no trump, it is won by the highest card of the suit led to it. [17]

Strictly speaking example (49) is false. However, the context in which (49) occurs might speak in its favour. ${ }^{15}$

(4) According to the analysis sketched above an indefinite in an only if-clause must be non-focal (topical, non-novel) in order to allow an anaphoric element in the main clause to pick up its possible values. The indefinite must contribute to the domain of quantification. This prediction is by and large confirmed. ${ }^{16}$

(5) Upon our analysis it ought to be possible to reformulate only if $A B$-sentences systematically as $B$, only if $A$. Indeed this turns out to be generally possible. The possibility to swap the two clauses is only blocked, systematically, if the $A$-clause contains an indefinite and the $B$-clause a related pronoun or anaphoric definite. Anaphoric relationships can thus be seen to systematically require an ante-cedent, and not to be satisfied with a post-cedent. For, indeed, if we swap the only if $A$ and the $B$-clause in these sentences, and at the same time exchange antecedents and anaphors, the results turn out fine again. Try for yourself, for instance, with the following examples:

(50) Only if a student changes residence to living at home or vice versa will the student need to contact the Financial Aid Office. [12]

(51) Only if a child fells right can he think right [27] 
(52) Only if a judge were actually biased should her decision be reversed. [80]

The fact that indefinites (deemed topical) can be so easily moved from the subordinate clause to the main clause may serve as a further indication that their semantic contribution is not local, and that, as we claim, it is projected into the domain of quantification. ${ }^{17}$

(6) In line with observations in (Geis 1973; McCawley 1981; Horn 1996, among others), a sentence Only if $A B$ must be (up to) equivalent with a contraposed sentence If not $A$ not $B$. The examples in our corpus support this thesis, but with one systematic qualification. Indefinites in the subordinate only if $A$-clause which are deemed topical naturally escape the negation in the if not $A$-clause. Try for yourself, with, e.g.:

(53) Only if a student's behavior is disruptive or coercive should it be prohibited. [21]

(54) Only if a U.S. exporter sells to the Nigerian government through an agent is there a registration fee requirement. [66]

Non-topical indefinites, however, do not do so:

(55) Only if a common framework is created, can innovative tax concepts be successful. [93]

(56) Only if a king's son would agree to marry me as a frog could the spell be broken. [105]

These observations thus give further support for the thesis that the semantic contribution of topical indefinites is not local. ${ }^{18}$

(7) Just for the record we mention that our corpus contains seven examples with a conjunctive if-clause such as (57), and four with a disjunctive one (like (47) above).

(57) Only if a student has participated and turned in work for more than half of the class will an "I" be given. [53]

All of these example seem to behave as can be expected. That is, they all seem to validate the following Boolean scheme:

(58) Only if $A$ and/or $A^{\prime} B \Leftrightarrow$ If not $A$ or/and not $A^{\prime}$, not $B$

But even though they are generally well-behaved, it can be difficult to choose the right Boolean - as can be seen from the con-disjunction:

(59) Only if a couple posted bond, obtained a license, and or presented a certificate to the county official would their marriage be recorded. [22]

Resuming our results so far we find that (i) only if-clauses indeed appear to state necessary conditions for possible states or events reported in the main clauses; (ii) topical (or non-novel) indefinites in these clauses project their semantic contribution into the relevant domains of quantification; and (iii) pronouns in these structures may be anaphoric upon antecedent indefinites in them if, and only if, these indefinites are topical. Up to this point (our interpretation of) the examples in our corpus support the views we have exposed above. 


\section{Causal and Other Dependencies}

Upon our basic analysis, "if" and "only if" are each other's (logical) converses, and they report regularities at best, no dependencies, and they are therefore deemed to be each other's converses. However, it has often been observed that conditionals are used to express dependencies between eventualities reported in main and subordinate clauses, and that this hampers a reformulation of only if $A B$ into $A$ if $B$, and vice versa. Indeed "if" and "only if" are not at all ideal converses in natural language. A stunning counterexample comes from (McCawley 1974; McCawley 1981):

(60) If butter is heated, it melts.

(61) *Butter is heated, only if it melts.

Whereas (60) seems a perfectly natural thing to say, and to be true, probably, its converse (61) sounds very odd, if indeed understandable at all.

The same holds for the majority of examples in our little corpus. Only if $A B$ and $A$ if $B$ do not seem to express exactly the same states of affairs, except in only a few cases:

(62) Only if a unit is flawless is it ready for shipping. [59]

(63) Only if a file is available at both sites the mirror sites are listed. [91]

(64) Only if a great saint occurs, do we permit [a relaxation] on his memorial [129]

(Note: the pronoun "his" in (64) is not anaphoric upon "a great saint".)

There are a few points to be made here. First, the observations about McCawley's (60-61) can be explained by a principle stated in (Talmy 1978; Reinhart 1984 ) according to which causal consequences of events reported in a main clause are not allowed to figure in subordinate clauses. ${ }^{19} \mathrm{~A}$ consequential interpretation of (60) is thus blocked in (61). Instead, (61) can be taken to suggest that, for butter, melting is a necessary condition for being heated, which, indeed, is an odd thing to state. $^{20}$

Second, the Talmy/Reinhart's principle only serves as an explanation of the non-convertibility of Only if $A B$ into $A$ if $B$ if these sentences report a 'deeper' connection between the material described in the main and the subordinate clauses than just a regularity or corelation. This point can be strengthened with an example from (von Fintel 1997). A reformulation of only if with if is possible if must is added (and will is dropped):

(65) We will celebrate only if John wins.

(66) ? If we will celebrate, John wins the race.

(67) If we celebrate, then John must have won the race.

According to (65) John's success is a prerequisite for a celebration, and this dependency blocks a reformulation into (66)-which even suggests that a possible 
celebration would speed up John sufficiently. But such a consequential relation can be canceled by adding an epistemic must, indicating that a possible celebration (as referred to with 67) constitutes sufficient evidence for conclusions about the outcomes of the race. Notice, however, that there is a subtle difference between (65) and (67). Whereas the first indeed seems to state a genuine dependency between possible events described by the main and the subordinate clause (a prerequisite, for instance), the second at best states a consequence from the dependency reported by the first, and not a dependency itself. A completely similar pattern can be observed with quite a few examples in our corpus, as you may try for yourself on:

(68) Only if a youngster is playing along with senior cricketers, he will develop in confidence and do well. [114]

(69) Only if a system also talks his language, a user will be convinced by machinefound proofs and feel his understanding of the topic improved. [123]

A major question at this point then is the following. If (only) if-sentences are used to report something over and above mere regularities, is this a uniform feature, and should it be taken to belong to the meaning of "(only) if"? Unfortunately, there is no room here to really go into this question, but we will not withhold from the reader the impression we have got from the examples in our corpus. These are, first, that there is a great variety of modals ${ }^{21}$ in the main clauses of our examples, and that these contribute to the expression of a great variety of dependencies. Many examples are concerned with regularities, necessities and possibilities of all kinds, personal, conventional, logical, moral, juridic, causal, .... Quite a few others are concerned with permission, obligation, advice and instruction, including that of the workings of machines and politicians. And although quite a lot of this variety can be attributed to the fact that conditionals may relate to a variety of "modal bases" (Kratzer 1981), it does not seem that they use these modal bases in a uniform way.

I am thus tempted to conclude, fully in the spirit of (Grice 1989), that there must be a uniform, basic meaning of "if" and "only", and that, as a matter of fact, this must be the weakest one, the one we have also employed in this paper. Additional aspects of meaning should be explained as the result of a combination of factors: on the one hand, the basic semantics of "only", "if" and of modal and temporal operators in the main clause of a conditional, and, on the other, relevant pragmatic features, such as information structure, implicit questions under discussion, indexical features, besides general pragmatic principles and constraints like those of Talmy and Reinhart.

\section{Conclusion}

This paper started out with the observation that inverse and only if-donkey sentences pose a problem for a classical dynamic semantic framework. We have sketched an account of these sentences in what we call a "second generation" framework, which crucially employs the notion of information developed in the first, but with a more classical and more flexible combinatorics. 
The main idea has been that donkey indefinites in inverse, only if and ordinary donkey sentences are topical and are projected into the domains of quantification of (im- or explicit) adverbial or adsentential quantifiers. The effects of this have been modeled on the basis of a classical analysis of "if" and "only" and a Karttunen and Peters-style treatment of information structure.

In passing we have argued that domain restriction effects of if-clauses are real, that these effects can, but need not, be attributed to the semantic contribution of if, and that if-clauses do not restrict nominal quantifiers (although their presuppositions may do so).

We have next tested our findings on a corpus of only if-donkey sentences drawn from the internet. Our intuitions about these examples indeed constitute motivation for the idea that topical indefinites do not semantically contribute to their local context, and that only these can be antecedents for anaphoric pronouns.

We also find strong motivation for the idea that if-and only if-sentences report something over and above the statement of a mere regularity, but we have not found sufficient reason to attribute this additional aspect of meaning to the meaning of "if" and "only". (Of course, not being able to find such a reason may be a shortcoming of the author.)

Just to conclude, let me repeat that our little corpus can be inspected at:

- http: //www.wins . uva.nl/ pdekker/SALT/

There you can also find some of the minimal context of our final number of the show:

(70) Only if a building is selected, and a name is picked randomly from a list of the persons in that building, can we use the fact that the name denotes a person on the 2 nd floor to infer that the probability that it is a 100-persons house is less than 75\%. (In fact, it's 8\%.) [13]

\section{Endnotes}

${ }^{1}$ This is also a characteristic feature of an E-type interpretation of pronouns.

${ }^{2}$ Notice that an E-type pronoun approach faces essentially the same problem.

${ }^{3}$ For the purposes of exposition it is the most transparent one to use here. When it actually comes to the study and motivation of the underlying semantics, we however prefer the system called 'predicate logic with anaphora' (PLA). For a technical introduction to that system, cf., (Dekker 2001); for more philosophical motivation, cf., (Dekker 200xb). The central ideas and properties of the systems $P L D C$ and $P L A$ are by and large the same.

${ }^{4}$ Notice that these four connectives are interdefinable, for, e.g., $\supseteq=(\lambda P \lambda Q Q \subseteq P)$, $\rightarrow=(\lambda p \lambda q(\lambda x p) \subseteq(\lambda x q)), \subseteq=(\lambda P \lambda Q \forall x(Q(x) \leftarrow P(x)))$, etc.

${ }^{5}$ Examples such as these are also discussed in (Bosch 1983).

${ }^{6}$ If the if-clause of (21) is given wide scope, it says that if Derek works hard, then only he will succeed. So it is part of his unchummy ambitions then to become the one and only who succeeds. 
${ }^{7}$ As (von Fintel 1998) observes, example (23) indeed corresponds to the examples figuring in what has been called "Peirce's puzzle". But sure enough, Peirce's puzzle is definitely not solved by a domain restriction analysis of if-clauses, but by a combined semantic pragmatic (Gricean) analysis of utterances of conditionals, completely similar to the one offered by Peirce himself. (See Dekker 200xa for discussion).

${ }^{8}$ As before, the suggested connection between main and subordinate clause seems to be stronger than the one given by a material implication analysis, but this is something which can be accounted for in pragmatic terms, as (Grice 1989) has already argued for. An essentially similar account can be given of the fact that quantification in (18) seem to be restricted to a domain of letters which are still under consideration for being answered.

${ }^{9}$ Notice that we could have done the same without the ${ }^{\wedge}$ 's and ${ }^{\vee}$ 's, but the present formulation may be more insightful.

${ }^{10}$ If if-clauses are analyzed as (incomplete) conditionals, there is indeed an arrow $\rightarrow$ present in the interpretation of the full sentences, contrary to the conclusion of (Kratzer 1991) quoted above. However, the point, entirely consistent with her conclusion, is that $\rightarrow$ doesn't really act as a two-place operator then. For, pushing the point a bit, such sentences can be analyzed as $O(p \rightarrow)(q)$, with $O$ the main (explicit or implicit) sentential operator. The whole issue, thus, trivializes.

${ }^{11}$ What we here envisage is, arguably, ${ }^{*}$ the* semantic correlate of the very well-behaved structural theory of (van der Sandt 1989; Geurts 1999). Indeed, using Heimian information sets, or discourse representation structures like Geurts and van der Sandt do, the so-called 'binding problem', which has been deemed so disastrous for Karttunen and Peters' analysis, automatically disappears. As (Karttunen and Peters 1979) themselves observed their analysis has a problem with the following example:

(71) Someone managed to succeed George V on the throne of England.

Upon Karttunen and Peters' analysis the person who is presupposed to have had difficulties with succeeding George V can be different from the one who is asserted to have done so eventually. But as soon as one can render the semantics of anaphoric relationships in order, this problem dissolves completely. For instance, example (71) can be said to presuppose that someone has tried hard to succeed George V, and to assert that that person eventually did succeed him.

${ }^{12}$ If $Q_{D}(A)(B)$ is a quantified construction evaluated relative to a domain $D$, and if $C$ is a presupposition of $A$ or $B$, then the whole can, in principle, be interpreted in one of two ways: as $Q_{D}(C \cap A)(B)$ or as $Q_{D \cap C}(A)(B)$. Distinguishing cases involve (readings of) determiners which do not satisfy extension or conservativity.

${ }^{13} \mathrm{We}$ here have to add a note on so-called asymmetric quantification. If indefinites are non-topical, as in:

(72) If a man [had a dime $]_{F}$, he threw it in the parking meter.

our analysis does not apply. However, there are independent reasons to assume that the pronoun "it" must act like some kind of an E-type pronoun in these contexts. Cf. (van Rooy 1997) for relevant discussion.

${ }^{14}$ An overview of the examples, in context, and with address, can be found at

- http://www.wins.uva.nl/ pdekker/SALT/

References to the examples in that file are given here in square brackets: '[nn]'. 
${ }^{15}$ There can be, and actually has been, discussion about the proper evaluation of example (49). I thank Ede Zimmermann for some pertinent comments.

${ }^{16}$ Only in seven cases the indefinite does not itself restrict the domain of quantification, but in six of these cases the indefinite is partly topical or functional upon the material that is quantified over, as, e.g., in (73):

(73) Only if a land worthy of economic exploitation was found would it be colonized. [124])

Only (74) seems to present a real counterexample:

(74) Only if a leader sees you he'll ask you if your in a server he's in. [49])

But then, indeed, it seems hard to make sense of this mysterious example. The context of (74) does not help.

${ }^{17}$ Swapping the two clauses of only if-sentences with indefinites that are partly topical requires some more subtle adjustments. Example (73), for instance, would have to be reformulated as:

(75) A land found would be colonized only if it was worthy of economic exploitation.

${ }^{18}$ There are also some interesting interactions between modals in the main $B$-clause and the second negation in the contraposed reformulation. Unfortunately, there is no room to go into this issue here.

${ }^{19}$ I am indebted to Antje Roßdeutscher for supplying me with the reference to Reinhart's work. Talmy states a principle like this as a possible universal of natural language.

${ }^{20}$ There are more constructions which may occur happily in a main clause, but not in subordinate clauses. Questions, demands, epistemic modals and probabilities can be conditionalized, whereas they are not allowed to figure in if-clauses. Consider:

(76) Do not invite the Smiths, if you don't like gossip.

*Only if do not invite the Smiths, you don't like gossip.

(77) If I agree, then what's in it for me?

${ }^{*}$ I agree, only if what's in it for me?

(78) If Mary is home, then maybe/probably Bill is around as well. ${ }^{*}$ Mary is home, only if maybe/probably Bill is around (as well).

${ }^{21}$ There are 28 examples which come without a modal or temporal operator, 34 with will, 20 with would, 17 with can, 3 with could, 9 with should, and 22 with modalities stated otherwise.

\section{Bibliography}

Aloni, M., Beaver, D., and Clark, B.: 1999, Focus and topic sensitive operators, in P. Dekker (ed.), Proceedings of the 12-th Amsterdam Colloquium, pp 55-60, ILLC/Department of Philosophy, Amsterdam

Bosch, P.: 1983, Agreement and Anaphora, Academic Press, London 
Dekker, P.: 1993, Transsentential Meditations. Ups and Downs in Dynamic Semantics, Ph.D. thesis, ILLC/Department of Philosophy, University of Amsterdam, Amsterdam

Dekker, P.: 1999, Scopes in discourse, Language and Computation 1(1), 7-32

Dekker, P.: 2000, The semantics of dynamic conjunction, in J. Seligman and P. Blackburn (eds.), Logic, Language and Computation, Vol III, pp 105-125, CSLI, Stanford, CA

Dekker, P.: 2001, Meaning and use of indefinite expressions, Journal of Logic, Language and Information 10, In print.

Dekker, P.: 200xa, Dynamics and pragmatics of "Peirce's Puzzle", Journal of Semantics, To appear

Dekker, P.: 200xb, Grounding dynamic semantics, in A. Bezuidenhout and M. Reimer (eds.), Descriptions and Beyond: An Interdisciplinary Collection of Essays on Definite and Indefinite Descriptions and other Related Phenomena, Oxford University Press, Oxford, Under review.

Gawron, J. M.: 1996, Quantification, quantificational domains and dynamic logic, in S. Lappin (ed.), The Handbook of Contemporary Semantic Theory, pp 247267, Blackwell, Oxford

Geis, M.: 1973, If and unless, in B. Kachru, R. Lees, Y. Malkiel, A. Petrangeli, and S. Saporta (eds.), Issues in Linguistics: Papers in Honor of Henry and Renée Kahane, pp 231-253, University of Illinois Press, Urbana

Geurts, B.: 1999, Presuppositions and Pronouns, Elsevier, Oxford

Grice, H.: 1989, Indicative conditionals, in P. Grice (ed.), Studies in the Way of Words, Harvard University Press, Cambridge, MA

Groenendijk, J. and Stokhof, M.: 1991, Dynamic predicate logic, Linguistics and Philosophy 14(1), 39-100

Heim, I.: 1982, The Semantics of Definite and Indefinite Noun Phrases, Ph.D. thesis, University of Massachusetts, Amherst, Published in 1988 by Garland, New York.

Higginbotham, J.: 1986, Linguistic theory and Davidson's program in semantics, in E. le Pore (ed.), Truth and Interpretation. Perspectives on the Philosophy of Donald Davidson, pp 29-48, Reidel, Oxford

Horn, L. R.: 1996, Exclusive company: Only and the dynamics of vertical inference, Journal of Semantics 13(1), 1-40

Jäger, G.: 1996, Only updates. On the dynamics of the focus particle only, in M. Stokhof and P. Dekker (eds.), Proceedings of the Tenth Amsterdam Colloquium, pp 387-405, ILLC, University of Amsterdam, Amsterdam

Kamp, H.: 1984, A theory of truth and semantic representation, in J. Groenendijk, T. Janssen, and M. Stokhof (eds.), Truth, Interpretation and Information, Foris, Dordrecht

Kamp, H. and Reyle, U.: 1993, From Discourse to Logic, Kluwer, Dordrecht

Karttunen, L. and Peters, S.: 1979, Conventional implicature, in C.-K. Oh and D. A. Dinneen (eds.), Syntax and Semantics 11 - Presupposition, pp 1-56, Academic Press, New York

Kratzer, A.: 1981, The notional category of modality, in H.-J. Eikmeyer and H. 
Rieser (eds.), Words, Worlds and Contexts, pp 38-74, de Gruyter, Berlin

Kratzer, A.: 1991, Conditionals, in A. von Stechow and D. Wunderlich (eds.), Semantik: ein internationales Handbuch der zeitgenössischen Forschung, pp 651-656, Walter de Gruyter, Berlin

Krifka, M.: 2001, Non-novel indefinites in adverbial quantification, in C. Condoravdi and G. R. de Lavalette (eds.), Logical Perspectives on Language and Information, CSLI Publications, Stanford

Lewis, D.: 1975, Adverbs of quantification, in E. L. Keenan (ed.), Formal Semantics, Cambridge University Press, Cambridge, U.K., 3-15

McCawley, J. D.: 1974, If and only if, Linguistic Inquiry 5, 632-635

McCawley, J. D.: 1981, Everything that Linguists have always Wanted to Know about Logic** but were ashamed to ask, Basil Blackwell, Oxford

Reinhart, T.: 1984, Principles of Gestaltperception in the temporal organization of narrative texts, Linguistics 22, 779-809

Stalnaker, R.: 1978, Assertion, in P. Cole (ed.), Syntax and Semantics 9 - Pragmatics, pp 315-332, Academic Press, New York

Stalnaker, R.: 1998, On the representation of context, Journal of Logic, Language and Information 7, 3-19

Talmy, L.: 1978, Figure and ground in complex sentences, in J. H. Greenberg, C. A. Ferguson, and E. A. Moravcsik (eds.), Universals of Human Language, pp 625-649, Stanford University Press, Stanford

van der Does, J.: 1996, An E-type logic, in J. Seligman and D. Westerståhl (eds.), Logic, Language and Computation, pp 555-570, CSLI, Stanford

van Eijck, J.: 2001, Incremental dynamics, Journal of Logic, Language and Information 10, To appear

van der Sandt, R. A.: 1989, Presupposition and discourse structure, in R. Bartsch, J. van Benthem, and P. van Emde Boas (eds.), Semantics and Contextual Expression, pp 267-294, Foris, Dordrecht

van Rooy, R.: 1997, Descriptive pronouns in dynamic semantics, in P. Dekker, M. Stokhof, and Y. Venema (eds.), Proceedings of the Eleventh Amsterdam Colloquium, pp 265-270, ILLC, University of Amsterdam, Amsterdam

Veltman, F.: 2001, How Natural is Natural Deduction for DPL?, Technical report, ILLC, Amsterdam

von Fintel, K.: 1994, Restrictions on Quantifier Domains, Ph.D. thesis, University of Massachusetts, Amherst

von Fintel, K.: 1997, Bare plurals, bare conditionals, and only, Journal of Semantics $14,1-56$

von Fintel, K.: 1998, Quantifiers and if-clauses, The Philosophical Quarterly 48, 209-214

Westerståhl, D.: 1984, Determiners and context sets, in J. van Benthem and A. ter Meulen (eds.), Generalized Quantifiers in Natural Language, pp 45-71, Foris, Dordrecht

Zeevat, H.: 1989, A compositional approach to discourse representation theory, Linguistics and Philosophy 12, 95-131 